\title{
Paediatric crossword puzzle - 27 (Answers)
}

Manouri P. Senanayake ${ }^{1}$

Sri Lanka Journal of Child Health, 2015; 44(3): 179

\begin{tabular}{|c|c|c|c|c|c|c|c|c|c|c|c|c|c|}
\hline & ${ }^{1} \mathrm{P}$ & & & ${ }^{2} \mathrm{H}$ & & ${ }^{3} \mathrm{M}$ & $C$ & $V$ & & & & & \\
\hline \multirow[t]{8}{*}{${ }^{4} \mathrm{~S}$} & 0 & 1 & $\mathrm{~L}$ & I & $\mathrm{N}$ & G & & & $B$ & 0 & ${ }^{6} \mathrm{~N}$ & D & \\
\hline & $\mathrm{N}$ & & & V & & & & & & & 0 & & \\
\hline & $\mathrm{T}$ & & ${ }^{7} \mathrm{R}$ & & & & & ${ }^{8} \mathrm{P}$ & & ${ }^{9} \mathrm{P}$ & D & D & \\
\hline & 1 & & $E$ & & ${ }^{10} \mathrm{D}$ & & & $\mathrm{R}$ & & & A & & \\
\hline & ${ }^{11} \mathrm{~N}$ & $\mathrm{U}$ & $\mathrm{T}$ & $\mathrm{R}$ & I & $\mathrm{T}$ & 1 & 0 & $\mathrm{~N}$ & ${ }^{12} \mathrm{~A}$ & $\mathrm{~L}$ & & \\
\hline & $E$ & & I & & $E$ & & & $P$ & & $\mathrm{~L}$ & & & \\
\hline & & & $N$ & & ${ }^{13} \mathrm{~T}$ & ${ }^{14} S$ & & 0 & & P & & & \\
\hline & & & 0 & & & I & & $F$ & & $\mathrm{H}$ & & ${ }^{5} \mathrm{~A}$ & \\
\hline \multirow[t]{2}{*}{${ }^{16} \mathrm{G}$} & I & $\mathrm{R}$ & $\mathrm{L}$ & ${ }^{17} \mathrm{~S}$ & & $D$ & & 0 & & ${ }^{18} \mathrm{~A}$ & I & $\mathrm{R}$ & $Q$ \\
\hline & & & & $\mathrm{M}$ & & $\mathrm{D}$ & & $\mathrm{L}$ & & & & V & \\
\hline \multirow[t]{4}{*}{${ }^{19} \mathrm{D}$} & ${ }^{20} \mathrm{E}$ & $L$ & $\mathrm{~T}$ & A & & $\mathrm{H}$ & & & & & ${ }^{21} \mathrm{R}$ & & \\
\hline & B & & & L & & ${ }^{22} \mathrm{~A}$ & D & $23 \mathrm{~J}$ & $A$ & C & $E$ & $\mathrm{~N}$ & ${ }^{24} \mathrm{~T}$ \\
\hline & V & & ${ }^{25} \mathrm{E}$ & $\mathrm{L}$ & $F$ & & & 1 & & & $T$ & & 0 \\
\hline & & & & & & & & ${ }^{26} \mathrm{~A}$ & $P$ & A & $\mathrm{T}$ & $\mathrm{H}$ & $Y$ \\
\hline
\end{tabular}

${ }^{1}$ Senior Professor in Paediatrics, Faculty of Medicine, University of Colombo 